\title{
Escuela, trabajo y desigualdad en la era digital. Claves desde la formación de oficios digitales a sectores desaventajados
}

\author{
School, jobs, and inequality in the digital age. Keys \\ from the development of digital trades to disadvantaged \\ sectors
}

Tobeña, Verónica

Verónica Tobeña Sobre la autora

vtobena@flacso.org.ar

Instituto de Investigaciones Sociales de América Latina (IICSAL), Argentina

Itinerarios educativos

Universidad Nacional del Litoral, Argentina

ISSN: $1850-3853$

ISSN-e: $2362-5554$

Periodicidad: Anual

vol. 1, núm. 14, 2021

revistadelindi@fhuc.unl.edu.ar

Recepción: 15 Noviembre 2020

Aprobación: 26 Julio 2021

URL: https://doi.org/10.14409/ie.2021.14.e0009

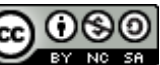

Esta obra está bajo una Licencia Creative Commons AtribuciónNoComercial-CompartirIgual 4.0 Internacional.
Resumen: La educación escolarizada es un dispositivo moderno que parte del diálogo con una época casi extinguida. La escuela secundaria, además, surge como una institución selectiva, destinada solo para algunos. En la Argentina los sectores populares pudieron acceder a este nivel incorporándose a circuitos de escolarización técnica, concebidos especialmente para ellos. Esta diferenciación se basa en la jerarquización de los saberes realizada por la modernidad, que distinguió teoría y práctica, destrezas intelectuales y manuales. La crítica a este modelo es de larga data, pero es con los profundos cambios epistemológicos, culturales y tecnológicos que conlleva la era digital que existe mayor consenso para repensar dicho proyecto formativo. En este marco han surgido una serie de ofertas de formación en oficios digitales orientadas a jóvenes de barrios populares que parecen refutar la tesis del capital cultural de origen trazadas por la sociología crítica hace medio siglo para la escuela. Este trabajo se propone auscultar dichas ofertas a la luz de ejes escolares que hoy están siendo revisados (uso de las TIC, propuesta pedagógica, relación con el conocimiento) para aportar evidencia que problematice el carácter epistemológico-pedagógico en el que se cifra la desigualdad que produce y reproduce la escuela.

Palabras clave: escuela secundaria , pedagogía , oficios digitales, sectores desaventajados.

Abstract: School education is a modern device that starts from a dialogue with an almost extinct era. Secondary school, moreover, emerges as a selective institution intended only for some. In Argentina, working-class teenagers were able to access this level by joining technical schooling programs especially designed for them. This distinction is based on the hierarchisation of knowledge outlined by modernity, which distinguished theory and practice, intellectual and manual skills. There is a long-standing criticism against this model, which has grown and led to greater consensus on rethinking this educational project as a result of the profound epistemological, cultural, and technological changes brought about by the digital era. In this context, a series of trainings in digital trades oriented to young people from working-class neighbourhoods have emerged and seem to refute the thesis of the cultural capital of origin traced by critical 


\begin{abstract}
sociology for schools half a century ago. By analyzing these offers in the light of the school core concepts that are currently under review (use of ICT, pedagogical proposal, relationship with knowledge), we provide evidence that might pose questions about the epistemologicalpedagogical character that leads to the inequality produced and reproduced by school.
\end{abstract}

Keywords: secondary school, pedagogy, digital occupations, disadvantaged sectors.

\section{Relación escuela desigualdad}

Siempre hemos vivido en un mundo desigual. La pregunta es cómo se produce esta transmisión de la condición desigual a través de las diferentes generaciones. Porque la transferencia se realiza a pesar de que los recursos en base a los cuales se construye el poder y la riqueza han variado a través del tiempo (Tiramonti y Tobeña, 2021). Nos encontramos en uno de esos momentos de reconfiguración de los factores asociados a la generación de riqueza y de reestructuración del sistema productivo, y con ello asistimos a la redefinición de cuáles son las actividades económicas más dinámicas. Entre ellas se recorta la «economía del conocimiento»; su potencial es enorme porque no se ciñe a un área o una rama de la actividad productiva sino que es transversal al conjunto de la economía, puesto que consiste en agregar valor a partir de replicar su existencia en el mundo digital y aprovechar la creación de esa interfaz para potenciar y expandir cada negocio. Su desarrollo en la Argentina es incipiente, está en ascenso y cuenta con pleno empleo, pero su crecimiento no alcanza el ritmo y la magnitud que podría debido a la falta de perfiles formados para los puestos vacantes.

¿Cómo podría la escuela formar estos perfiles? O, ¿qué le piden las empresas del conocimiento a la escuela?

La arena educativa oficial aún no ha respondido con políticas de envergadura a esta demanda insatisfecha. ${ }^{1}$ Sí han aparecido muchas ofertas de formación en diálogo con estos oficios digitales tanto del sector privado como del tercer sector. ${ }^{2}$ Nuestra intención aquí es interrogar dichas iniciativas a la luz de los ejes que hoy están siendo revisados en la escuela secundaria para poder profundizar la reflexión sobre ese nivel desde otros ángulos, otras experiencias que ayuden a repensarla.

\section{De los oficios manuales a los oficios digitales}

El primer gesto que se destaca de estas iniciativas, fundamentalmente las destinadas específicamente a grupos socialmente desfavorecidos, es que desafían el imaginario que asocia a estos sectores con su inserción económica desde oficios manuales. Los propios destinatarios de la propuesta dan cuenta del arraigo que tiene esta asociación y cómo deben desmontarla tanto en su medio social como en los nuevos entornos de trabajo a los que se integran:

No, flaco, acá no hay laburo digital. En este barrio tenés que ser mecánico como tu viejo (Leandro, Potrero Digital) 
A la gente le cuesta entender que vengas de un barrio humilde y te dediques a trabajos digitales. A veces cuando me junto con algunos colegas en la oficina, me tiran, 'La Matanza, ¿qué hacés acá?', como si vinieras de Marte (Leandro, Potrero Digital) Eso no es para vos. ¿Por qué no te haces un curso de costura? (Elizabeth, Potrero Digital)

El gesto de desnaturalizar las expectativas laborales construidas socialmente para estos grupos se concreta recortando un arbitrario cultural distinto y nuevo para ellos; uno que permite pensar en una propuesta de formación que parece escapar a la dinámica de reproducción de la desigualdad para apostar a producir nuevas oportunidades para este sector social. ${ }^{3}$

\section{Saberes tecnosociales}

Arbusta se autodefine como una empresa que brinda servicios de aseguramiento de calidad de software, datos e interacciones a grandes, medianas empresas y gobiernos en sus procesos de transformación digital. Su singularidad, nos explica su director Juan Umarán, «es que el talento que contrata Arbusta es un talento no mirado». Y agrega: «El lugar donde vivimos no determina nuestras capacidades ni nuestro potencial. El talento digital es universal». Consecuente con esta premisa, Abusta se propone desarrollar talento millenial que recluta entre jovenes de barrios populares sin formación ni experiencia previa y por los tanto con dificultades para conseguir trabajo formal. «En Arbusta estamos derrumbando un mito», reza el reporte de marzo de 2019 de la empresa, donde constan las más de 30 empresas que integran su cartera de clientes y guarismos que hablan de la buena marcha de la compañía, con sedes en Buenos Aires, Rosario, Medellín y Montevideo.

Podríamos analizar esta idea del «talento digital universal» de la que nos habla Umarán desde el cristal de la literatura que trabaja con los cambios en la constitución subjetiva a los que da paso la emergencia de las nuevas tecnologías de la información y la comuncación (TIC), apoyándonos entonces en conceptos como nativos digitales, Generación post-alfa (Berardi, 2010), Pulgarcita (Serres, 2013), Los Bárbaros (Baricco, 2008). Con sus matices y sus improntas singulares, esta literatura configura un marco teórico convergente en la idea de que de las subjetividades moduladas al calor de la era digital resultan otras cabezas. Que la tecnología (entendida como interfaz, como mediación que nos abre al dialogo con el mundo), tiene un papel central en el modo en que nos pensamos a nosotros mismos, en que pensamos la realidad, en que organizamos nuestras vidas, en que organizamos nuestra propia subjetividad, y el modo en que la sociedad se narra a sí misma (Peirone, 2020; Scolari, 2018). En definitiva, que el paso del libro a las tecnologías digitales tiene consecuencias trascendentales, que quedan bien resumidas en la identificación de este nuevo orden con la noción acuñada por Manuel Castells: sociedad informacional.

El ecosistema tecnológico en el que crecieron quienes hoy son jóvenes es muy diferente al entorno en el que crecimos nosotros, por eso su cabeza es otra.

Fernando Peirone (2018) bautizó con la categoría de saberes tecnosociales a esos conocimientos que se originan vinculados a la vida que los jóvenes desarrollan en el territorio digital. Esos saberes surgen en la vida online pero la rebasan, rebotan entre el mundo y el ultramundo digital, trazando un entramado que legítimamente podemos llamar la realidad (Baricco, 2019: 93). Hoy la realidad 
se transita en este doble circuito: es una suerte de cinta de Moebius que se desliza del espacio físico al virtual sin solución de continuidad. Según la investigación de Peirone, los jóvenes que trabajan en Arbusta, muchos de los cuales no completaron el nivel secundario,

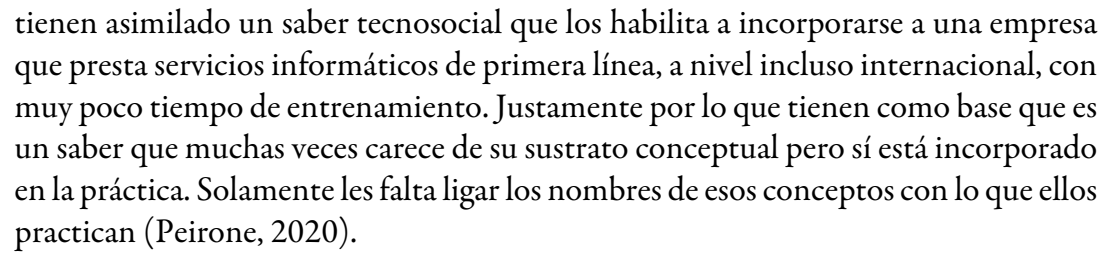
que presta servicios informáticos de primera línea, a nivel incluso internacional, con muy poco tiempo de entrenamiento. Justamente por lo que tienen como base que es un saber que muchas veces carece de su sustrato conceptual pero sí está incorporado en la práctica. Solamente les falta ligar los nombres de esos conceptos con lo que ellos practican (Peirone, 2020).

A diferencia de la lectoescritura que nosotros cultivamos en la escuela, estos jóvenes dominan saberes tecnosociales que forjan en la práctica social virtual, es decir, no son producto de un saber experto que adquieren con el método de «la letra con sangre entra» sino que lo dominan a fuerza de ensayo y error, de hacer y experimentar, de asumir retos y desafíos.

¿Cómo liga Arbusta esos saberes prácticos con los teóricos? ¿Cómo modela ese talento digital millenial, que reciben en estado bruto, para formarlos en un oficio digital? Su director nos explica cómo se instrumenta esta asimilación más conceptual de la que habla Peirone: «Como aprenden haciendo la explicitación del conocimiento no ocurre, es más tácita. Lo que trata Arbusta es de cultivar la conversación. Entendemos que la organización de Arbusta se basa en una madeja de conversaciones lo más explícita posible».

Si la educación escolar está enfocada en las reglas, en las convenciones y las formalidades que hay detrás de los usos de las matemáticas o la lengua, es decir, haciendo de las disciplinas un fin en sí mismo (Pérez Gómez, 2017; Frasca, 2012), en Arbusta el abordaje es inverso, porque parten del hacer y luego los exponen a los conceptos que condensan esos procesos involucrados en la tarea realizada para anclarlos teóricamente. Umarán nos explica que es un modelo de trabajo que se conoce como 'metodologías ágiles' y que es una dinámica estrechamente ligada a las posibilidades que brindan las herramientas digitales: «la idea es no esperar las grandes definiciones para hacer algo. Ir evolucionando en tiempos cortos, ser dinámico. Esta herramienta de trabajo está vinculada a las características de la tecnología .

Richard Sennett (2009) reconoce en este «aprender haciendo» una forma de aprendizaje propia del artesanado, interrumpida por la secularización que introdujo la tradición cultural moderna, que llevó a diferenciar y separar la teoría de la práctica. En cambio, en el contexto de comunidades de artesanado digitales se observa el cultivo de otra tradición, heredera del trabajo en talleres y que podría resumirse con la tesis a favor de la cual Sennet escribe el libro el artesano: «hacer es pensar» (Sennett, 2009: 9).

Arbusta da cuenta de un modelo de trabajo afín al que describe Sennet, porque en ella, como en aquellas comunidades de práctica que nucleaban a los artesanos, hay implícita una teoría del aprendizaje que integra tanto la importancia de la experiencia como fuente de aprendizaje como el constructivismo social: «la interacción refuerza y evidencia el carácter constructivista de las estrategias que ponen a jugar los y las jóvenes, ya que necesariamente hay una experiencia acumulada propia y de los demás gravitando en todo el proceso» (Bordignon et.al., 2020: 9). 


\section{IV. ¿Qué pedagogía corresponde al desplazamiento de la cultura ilustrada a la cultura digital?}

La forma en que los jóvenes llegan a dominar dichos saberes tecnosociales puede compararse con la forma en la que aprenden a hablar, aunque esta experiencia no es comparable completamente al fenómeno de la lengua materna porque son huérfanos digitales, prefiguran su cultura (Mead, 1997) de forma autodidacta, interactuando en redes, entre pares y en comunidades de práctica (Wenger, 2014). Pero al igual que con el habla, aprenden asumiéndose como productores y no como meros consumidores de cultura (Tobeña, 2020a y 2020b).

Para ello se vuelcan en general a las narrativas audiovisuales y se deslizan por diferentes lenguajes, porque los nuevos medios ya no los obligan a ser lineales, a permanecer anclados en un lugar mental. Alessandro Baricco lo explica muy bien en The Game: lo que les permite la Web es «dejarse dictar por el mundo la estructura de sus pensamientos y los movimientos de su mente» (2019: 87). Los soportes digitales median una representación del conocimiento que supera la cultura analógica y la abstracción que esta conlleva porque rompe con el carácter formal del saber para pensarlo de forma encarnada (Tobeña, 2020c).

Ahora bien, la escuela enseña a partir de los saberes pedagógicos que dialogan con la cultura de la ilustración (como los saberes de las disciplinas y sus didácticas generales y específicas) y necesitamos entender cómo conquistan los saberes tecnosociales los jóvenes para complementar dichos conocimientos para la enseñanza con nuevos conocimientos pedagógicos que dialoguen con la cultura digital.

$\mathrm{Al}$ respecto Peirone aporta más precisiones de cómo transcurre ese proceso que les permitió este aprendizaje a los jóvenes:

\footnotetext{
tuvieron que crear lo que nosotros llamamos una suerte de zona de desarrollo próximo sin un saber maduro que traccionara ese saber latente. Sino que es puro saber latente, interactuando y traccionándose mutuamente para la consecución de objetivos y la consecución de cuestiones vinculadas a lo que necesitaran saber (Peirone, 2020).
}

Hasta aquí el método, pero ¿qué hay del sustrato, de la materialidad de ese saber, de su naturaleza específica? Sobre esto Peirone suma una reflexión que señala la vacancia pedagógica que emerge con la cultura digital:

\footnotetext{
El problema es que se trata de un saber que hasta el momento no se ha podido subordinar a palabras, no se ha podido subordinar a una discursividad. Se trata de un saber que de alguna manera se desplaza del logos hacia el ícono, se desplaza de la lógica escritural hacia una lógica audiovisual. Y por lo tanto tiene una lógica de transmisión y de asimilación completamente diferente. Y por lo tanto tiene una lógica de enseñanza diferente (Peirone, 2020).
}

Una de las hipótesis de Arbusta es que hay muchas formas de aprender y por eso decidieron borrar de su vocabulario la noción de capacitación. Sus cuadros manageriales convinieron en que ellos mismos aprendieron haciendo y que esta forma de crecer tenía que tener un correlato en el repertorio de experiencias que promueva la cultura de la empresa. En la voz de Umarán, su estrategia queda fundamentada del siguiente modo: 
Si hacemos un inventario de las distintas formas de aprender hay algunas más fáciles de anotar que otras, unas más fáciles de saber que ocurrieron y otras menos. Aprender del feedback, del cliente, del error, de los compañeros, del curso de inglés, etc. Cada persona dentro de Arbusta tiene un recorrido de qué va aprendiendo y tiene que ver con los diferentes desafíos a los que se va sumando, de menor a mayor complejidad.

El especialista en tecnopedagogía Alejandro Artopoulos, también haciendo alusión a la experiencia de Arbusta, aporta una reflexión convergente con las miradas desplegadas por Peirone y Umarán:

la prioridad de la abstracción ya no es una regla. No se puede priorizar lo abstracto o lo concreto, se trata de ir y venir de uno a otro y sin jerarquías. Esto es gracias al saber computacional que reestructura todos los saberes. Rearma las prioridades didácticas y pedagógicas (2020).

Incorporar esta nueva forma de habitar el mundo que conlleva la cultura digital es el gran desafío de la educación argentina. Implica revisar el proyecto formativo que heredó de las sociedades disciplinarias que encuadraron a los sistemas educativos modernos. En este punto vale recordar que hasta ahora lo que viene organizando el trabajo en la escuela es la disciplina: el curriculum se ordena por asignaturas, pero también el orden escolar se tramita en base a una disciplina formal, que se agota en la toma de asistencia, en el cumplimiento de horarios y en rituales de obediencia.

Hoy necesitamos organizar el trabajo por problemas, por proyectos, desde abordajes interdisciplinarios. Necesitamos superar la pedagogía de la enunciación en pos de una pedagogía de la participación (Piscitelli, 2009), que piense a la escuela como un lugar para la exploración con el conocimiento, que se apoye en las tecnologías digitales para potenciar sus posibilidades y que pueda redefinir los espacios y los tiempos para el trabajo en articulación con las necesidades de la tarea que se tiene entre manos. Una escuela que se piense como un laboratorio, que enseñe a estar juntos aprendiendo colaborativamente, permitiendo el desarrollo de capacidades individuales en el marco de un trabajo colectivo. La disciplina escolar se resignifica en cómo aborda el gobierno de la conducta, ahora debe atender al desarrollo de habilidades blandas relacionadas con el procesamiento de las emociones y de los conflictos que surgen de la vida y el trabajo en equipo. ${ }^{4}$ Debe, a su vez, incentivar la autonomía de los alumnos en el desarrollo de su tarea y proporcionar las condiciones para la creatividad y el surgimiento del pensamiento lateral o divergente (Tiramonti y Tobeña, 2021).

Esta forma de organizar el trabajo escolar tiene como correlato un cambio en la cultura de trabajo del docente, que fue formado en una disciplina, para impartir conocimientos y para trabajar de forma solitaria. Para los cuerpos docentes esta nueva forma de organizar el trabajo implica trabajar en comunidades de práctica, implica lo que Beatrice Ávalos (2011) denomina como desprivatizar la práctica.

\section{V. ¿Fin de la tesis del capital cultural?}

$\mathrm{Al}$ acuñar la noción de saberes tecnosociales el investigador Fernando Peirone trabaja a la luz de una hipótesis: que estos saberes son transclasistas, transnacionales y transculturales y por lo tanto «la idea de capital cultural hoy podría estar perfectamente en discusión» (Peirone, 2020). 
Arbusta brinda argumentos a favor de esa hipótesis. De modo que podemos ver a Arbusta como un botón de muestra. Muestra de que es posible tener un origen social vulnerable, una escolarización trunca y carecer de experiencia laboral, y al mismo tiempo ser capaz de integrarse a un empleo formal en uno de los sectores del mercado laboral más dinámicos de la economía. Arbusta es muestra, entonces, que en la era digital el capital cultural de origen puede no constituir un obstáculo para establecer un diálogo activo con la cultura.

¿Hay en el declive del arbitrario cultural ilustrado que materializa la expansión de la cultura digital una oportunidad para que la educación escolar se reposicione como una institución emancipadora? ¿Podría la escuela aprovechar el cambio cultural contemporáneo para redefinir su proyecto formativo corrigiendo los sesgos socioculturales que cifraban su arbitrario cultural?

\section{Referencias}

Artopoulos, A. (2020). Plataformas de simulación y aprendizaje. Conversatorio Dossier Propuesta Educativa Nro. 53: Educación y Contemporaneidad. Buenos Aires: FLACSO. Recuperado de https://www.youtube.com/watch?v=ekggHnRabpw $\& \mathrm{t}=5624 \mathrm{~s}$

Ávalos, B. (2011). El liderazgo docente en comunidades de práctica. Educar vol. 47(2), 237-252.

Baricco, A. (2008). Los Bárbaros. Ensayo sobre la mutación. Buenos Aires: Anagrama.

Baricco, A. (2019). The Game. Buenos Aires: Anagrama.

Berardi, F. (2010). Generación post-alfa. Buenos Aires: Tinta Limón.

Bolivar, A. (2012). Justicia social y equidad escolar. Una revisión actual. Revista internacional de educación para la justicia social (RIEJS) volumen 1 (1), 9-45.

Bordignon, et al. (2020). "Exploración de las estrategias de aprendizaje tecnosocial entre los y las jovenes ingresantes a la educación superior”. Propuesta Educativa nro. 53, 9-24.

Frasca, G. (2012). Los videojuegos enseñan mejor que la escuela. TedxMontevideo. Recuperado de https://www.youtube.com/watch?v=TbTm1Lkm18o\&t $=2 \mathrm{~s}$

Mead, M. (1997). Cultura y compromiso. Barcelona: Gedisa.

Peirone, F. (2020). "Exploración de las estrategias de aprendizaje tecnosocial entre los y las jóvenes ingresantes a la educación superior. El caso UNIPE - UNPAZ UNSAM". En: Conversatorio Dossier Propuesta Educativa Nro. 53: Educación y Contemporaneidad. Buenos Aires: FLACSO. Recuperado de https://www.youtu be.com/watch? $\mathrm{v}=\mathrm{ekggHnRabpw \& t}=5624 \mathrm{~s}$

Peirone, F. (2018). El saber tecnológico. De saber experto a experiencia social. Virtualidad, Educación y Ciencia, 17 (9), 66-80.

Pérez Gómez, A. I. (2017). Pedagogias para tiempos de perplejidad. De la información a la sabiduría. Rosario: Homo Sapiens.

Piscitelli, A. (2009). Nativos digitales. Buenos Aires: Santillana.

Scolari, C. (2018). Las leyes de la interfaz. Barcelona, Gedisa.

Sennett, R. (2009). El artesano. Buenos Aires: Anagrama.

Serres, M. (2013). Pulgarcita. Buenos Aires: Fondo de Cultura Económica.

Tiramonti, G. y Tobeña, V. (2021). Políticas educativas para el mundo digital. Espacios en Blanco(serie indagaciones) Nro. 31. UNCPBA, pp. 309-325. Recuperado 
de https://ojs2.fch.unicen.edu.ar/ojs-3.1.0/index.php/espacios-en-blanco/articl e/view/938

Tobeña, V. (2020a). Repensar el futuro de la escuela desde comunidades de práctica. Claves desde TikTok. Dilemata Nro. 33, España, pp. 221-233. Recuperado de ht tps://www.dilemata.net/revista/index.php/dilemata/issue/view/33

Tobeña, V. (2020b). Gente Rota. Mestiza. UNAJ. Recuperado de http://revistamestiz a.unaj.edu.ar/gente-rota/

Tobeña, V. (2020c). La cuarentena como oportunidad para sintonizar la educación con la cultura contemporánea. Bitácora Educativa, Buenos Aires: FLACSO. Recuperado de https://www.ecys.flacso.org.ar/post/en-cuarentena-y-conectados -qué-nuevo-paradigma-educativo-sugiere-el-mundo-digital

Wenger, E. (2014). Communities of practice: a brief introduction. Recuperado de https://wenger-trayner.com/wp-content/uploads/2013/10/06-Brief-introdu ction-to-communities-of-practice.pdf

\section{Notas}

1 Entre las políticas que para destacar están las Escuelas Experimentales PROA (Programa Avanzado de Educación Secundaria con énfasis en TIC) creadas en Córdoba en el 2014, o la Escuela de Robótica de Misiones, creada en el 2017. Luego existen planes y/o programas de formación de iniciativa estatal para la formación en distintos oficios digitales como el Programa 111 mil, Codo a Codo 4.0, Aprendé programando, entre otros.

2 Centros como Digital House, Coder House, Acámica, Escuela Da Vinci y Rolling Code, entre otros, son exponentes de esta tendencia para el público de sectores medios y medios/altos. En el caso de las ofertas destinadas a sectores desfavorecidos, puede mencionarse, entre muchos otros, a Potrero Digital en la Provincia de Buenos Aires, Puerta 18 en CABA, a la Fundación León/Infomanager en Tucumán, y a la empresa Arbusta, una compañía que brinda servicios digitales y para ello emplea jovenes de sectores vulnerables a los que no les exige título secundario y se ocupa de capacitarlos y formarlos en las habilidades técnicas y blandas necesarias para desempeñarse en este sector. Este ensayo se elabora en base, fundamentalmente, al caso de la empresa Arbusta, principal referente empírico e informante de este estudio. El caso de Potrero Digital también constituye un insumo para este trabajo, aunque en menor medida.

3 Contamos desde los años sesenta y setenta con las investigaciones que desde la sociología de la educación indagan sobre ese papel que estaría jugando la escuela en la ecuación de la desigualdad. Estos estudios -los de Bourdieu y Passeron en Francia, de Basil Bernstein en Inglaterra y el Informe Coleman en Estados Unidos- dan cuenta de que, antes que productora de oportunidades, la escuela moderna es más bien reproductora de desigualdades. Dichos trabajos pusieron bajo la lupa el arbitrario cultural y el tipo de vínculo con el saber que promueve la pedagogía transmisiva escolar, identificando allí al corazón de su matriz discriminatoria (Bolivar, 2012).

$4 \quad$ Las experiencias aquí analizadas pueden ser inspiradoras al respecto: «Tenemos por ejemplo-consigna Umarán-talleres de inteligencia emocional, pero en lo que tiene que ver con la realización de sus tareas. La gestión de la inteligencia emocional para el trabajo en un proyecto».

\section{Notas de autor}

Sobre la autora

Dra. en Ciencias Sociales, FLACSO Argentina; Mg. en Sociología de la Cultura y Análisis Cultural, IDAES - UNSAM; Lic. en Ciencias de la Comunicación, UBA. Es investigadora asistente del CONICET con sede en el IICSAL-FLACSO, Programa Educación, Conocimiento y Sociedad. Docente a cargo del Curso de Posgrado (modalidad virtual): 'El futuro ya llegó pero está mal distribuido'. La escuela en la era 
Verónica Tobeña. Escuela, trabajo y desigualdad en la era digital. Claves desde la formación de oficios digitales a sectores desaventajados

digital (UNQ). Su línea de investigación principal está dedicada a analizar cómo interpelan los cambios culturales y tecnológicos a la escuela. 